\title{
RGG1, Involved in the Cytokinin Regulatory Pathway, Controls Grain Size in Rice
}

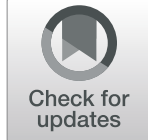

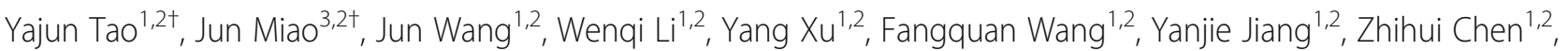
Fangjun Fan ${ }^{1,2}$, Mengbin $\mathrm{Xu}^{2,3}$, Yong Zhou ${ }^{2,3}$, Guohua Liang ${ }^{2,3^{*}}$ and Jie Yang ${ }^{1,2^{*}}$ (1)

\begin{abstract}
Heterotrimeric GTP binding proteins (G proteins) and cytokinin play important roles in regulating plant growth and development. However, little is known about the mechanism by which they coordinate the regulation of grain size in rice. We functionally characterized one gene, RGG1, encoding a type-A GY subunit. Strong GUS staining was detected in young panicles and spikelets, suggesting a role for this gene in modulating panicle-related trait development. Overexpression of RGG1 in Nipponbare (NIP) and Wuyunjing 30 (WYJ30) significantly decreased plant height, panicle length and grain length by regulating cell division. However, rgg1 mutants generated by the CRIS PR/Cas9 system exhibited no obvious phenotypic differences, which may be due to the extremely low expression level of this gene in vivo. The transcriptomes of young panicles of NIP, the NIP-rgg1-2 mutant and the NIP-OE2 overexpression line were sequenced, and the results showed that many differentially expressed genes (DEGs) were associated with the cytokinin biosynthetic pathway. We confirmed this result by measuring the endogenous cytokinin levels and found that cytokinin content was lower in the overexpression lines. Additionally, increased expression of RGG1 decreased sensitivity to low concentrations of 6-benzylaminopurine (6-BA). Our results reveal a novel $G$ protein —cytokinin module controlling grain size in rice and will be beneficial for understanding the mechanisms by which $\mathrm{G}$ proteins regulate grain size and plant development.
\end{abstract}

Keywords: Heterotrimeric G protein, RGG1, Rice, Grain size, Cytokinin

\section{Background}

Heterotrimeric GTP binding proteins (G proteins) are key regulators of a multitude of transmembrane signalling pathways in animals and plants. The heterotrimeric $G$ protein complex is composed of $G \alpha, G \beta$, and $G \gamma$ subunits, which cycle between active and inactive forms. G protein signalling is activated by seven-pass transmembrane $G$ proteincoupled receptors (GPCRs) that function as guanine nucleotide exchange factors and then transduce the signal to downstream effectors (Pandey 2019). In plants, G proteins are

\footnotetext{
*Correspondence: ricegb@yzu.edu.cn; yangjie168@aliyun.com

${ }^{\dagger}$ Yajun Tao and Jun Miao contributed equally to this work.

${ }^{2}$ Jiangsu Co-Innovation Center for Modern Production Technology of Grain Crops, Yangzhou University, Yangzhou 225009, Jiangsu, China

${ }^{1}$ Institute of Food Crops, Jiangsu Academy of Agricultural Sciences/Nanjing Branch of Chinese National Center for Rice Improvement, Nanjing 210014, Jiangsu, China

Full list of author information is available at the end of the article
}

involved in multiple fundamental growth and development pathways, including panicle branching (Huang et al. 2009; Zhou et al. 2009), seed size (Liu et al. 2018; Mao et al. 2010; Sun et al. 2018), shoot apical meristem (SAM) development (Bommert et al. 2013), nitrogen utilization (Sun et al. 2014), and stress tolerance ( $\mathrm{Yu}$ and Assmann 2015; Zhang et al. 2015).

Although G proteins are evolutionarily conserved, their numbers vary widely between humans and plants. For example, at least $23 \mathrm{G} \alpha, 5 \mathrm{G} \beta$ and $12 \mathrm{G} \gamma$ have been identified in humans. In contrast, the rice genome contains only one $\mathrm{G \alpha}$ (RGA1), one $\mathrm{G} \beta$ (RGB1), and five $\mathrm{G} \gamma$ homologs (RGG1, RGG2, GS3, qPE9-1/DEP1, and GGC2) (Sun et al. 2018). Mutation in RGA1 causes severe dwarfing and small grain size (Zong et al. 2018). Additionally, RGA1 is involved in regulating rice gibberellin and brassinosteroid signalling (Ueguchi-Tanaka 
et al. 2000; Wang et al. 2006). The G $\beta$ gene $R G B 1$ positively regulates cellular proliferation to modulate internode elongation and grain size (Utsunomiya et al. 2011). Evidence also shows that $R G B 1$ functions as a positive regulator of $A B A$ to modulate rice drought tolerance (Zhang et al. 2015). The five $\mathrm{G \gamma}$ proteins antagonistically regulate grain length in rice. In particular, GS3, qPE9-1/DEP1, and GGC2 competitively interact with $G \beta$ to control grain size (Sun et al. 2018). qPE9-1/DEP1 could directly interact with the MADS-domain transcriptional factor OsMADS1 and enhance its transcriptional activity to modulate grain size (Liu et al. 2018). RGG2, encoding a type B GB subunit, negatively regulates grain size and is also involved in gibberellin signalling (Miao et al. 2019). These prior studies in rice reveal that $G$ proteins play vital roles in the determination of grain size as well as in phytohormone regulation.

Phytohormones play diverse roles in plant growth and development (Blázquez et al. 2020). Cytokinin, one of the most important phytohormones, has been shown to modulate panicle traits. Gn1a encodes a cytokinin oxidase/dehydrogenase enzyme that is responsible for cytokinin degradation in vivo. Mutation in Gn1a results in cytokinin accumulation and causes an increase in grain number per panicle (GN) (Ashikari et al. 2005). Conversely, the cytokinin-activating enzyme LONELY GUY (LOG) directly converts inactive cytokinin to biologically active forms. The $\log$ mutant has highly reduced SAMs and panicles and abnormal branching patterns (Kurakawa et al. 2007). Cytokinin signalling plays important roles in regulating meristem cell proliferation and differentiation (Stahl and Simon 2010). Studies have shown that G proteins are also involved in stem cell fate determination. Maize COMPACT PLANT2 (CT2), which encodes a $\mathrm{G \alpha}$ subunit, interacts with FASCIATE EAR2 (CLV2) to regulate inflorescence meristem size (Bommert et al. 2013). In Arabidopsis, G $\beta$ mutants showed an enlarged meristem size (Ishida et al. 2014). AGB1 interacts with RPK2, one of the CLV3 peptide hormone receptors, to regulate meristem development. However, rice $\mathrm{G}$ proteins involved in CLAVATA signalling have not been reported.

Decreased levels of cytokinin also lead to reduced grain size in the root enhancer1 (ren1-D) mutant due to activation of OsCKX4 (Gao et al. 2014), and grain size may be regulated in part by modulation of long-distance transport of cytokinin by Big Grain3 (BG3), which encodes a purine permease, OsPUP4 (Xiao et al. 2019). Recently, the $\mathrm{G \gamma}$ subunit $q P E 9-1 / D E P 1$ was found to positively regulate grain filling by increasing endogenous cytokinin and auxin concentrations in rice grains (Zhang et al. 2019). However, how G proteins interact with cytokinin signalling to control growth and development in plants remains largely unknown.
In this study, we functionally analysed the $\gamma$-subunit gene RGG1 in rice. Overexpression of RGG1 caused reduced plant height and grain length. Further results suggested that RGG1 modulates endogenous cytokinin accumulation and responses to regulate plant morphology and grain development.

\section{Results}

\section{RGG1 Encodes a Type-A Gy Subunit}

In the rice genome, five Gy subunits have been identified. Among them, RGG1 is relatively small and contains four exons (Fig. 1a). Phylogenetic analysis showed that the G proteins of rice, Arabidopsis, and maize were divided into three groups (Fig. 1b). Among types A, B and C, the amino acid sequences showed very little conservation, and most of the similarities were limited to a highly conserved GGL (G gamma-like) domain (Fig. S1). RGG1 belongs to a clade of type-A G proteins along with the AGG1 and AGG2 proteins of Arabidopsis. SMART analysis predicted that $R G G 1$ contains a nuclear location signal (NLS) at the $\mathrm{N}$-terminus, a GGL domain, and a CaaX isoprenylation motif at the C-terminal end, typical of all canonical typeA G proteins (Fig. S1).

We confirmed that RGG1 interacts with RGB1 using a bimolecular fluorescent complementation (BiFC) assay. The BiFC fluorescence signal was detected in the membrane, cytoplasm and nucleus, suggesting the potential function of the $G \beta \gamma$ dimer (Fig. 1c). To further explore the interaction of RGG1 and RGB1, several truncated RGG1 proteins were generated. As shown in Fig. 1d, the GGL domain was necessary and sufficient for interaction with RGB1. In addition, residues 55-67 of RGG1 were required for the RGG1-RGB1 interaction (Fig. 1d).

\section{Expression Profiles and Subcellular Localization}

To determine the expression pattern of RGG1, the tissue-specific expression of RGG1 was detected using transgenic plants containing an RGG1 promoter: GUS fusion. GUS staining revealed different levels of expression of RGG1 in panicles at different developmental stages. As shown in Fig. 2a, the expression of RGG1 gradually decreased with panicle development. It was also expressed in roots, with particularly strong staining in the root tips (Fig. 2b). Additionally, GUS staining showed that RGG1 was abundantly expressed in leaves, sheaths, nodes, stems, and spikelets (Fig. 2c-h). Moreover, the GUS results were in agreement with our quantitative reverse transcription-PCR (qPCR) analyses, which showed particularly high expression of RGG1 in young panicles and decreasing panicle expression as development progressed (Fig. 2i). In addition, we detected RGG1 transcripts in other tissues using qPCR, including leaves, stems, nodes, sheaths, and roots 


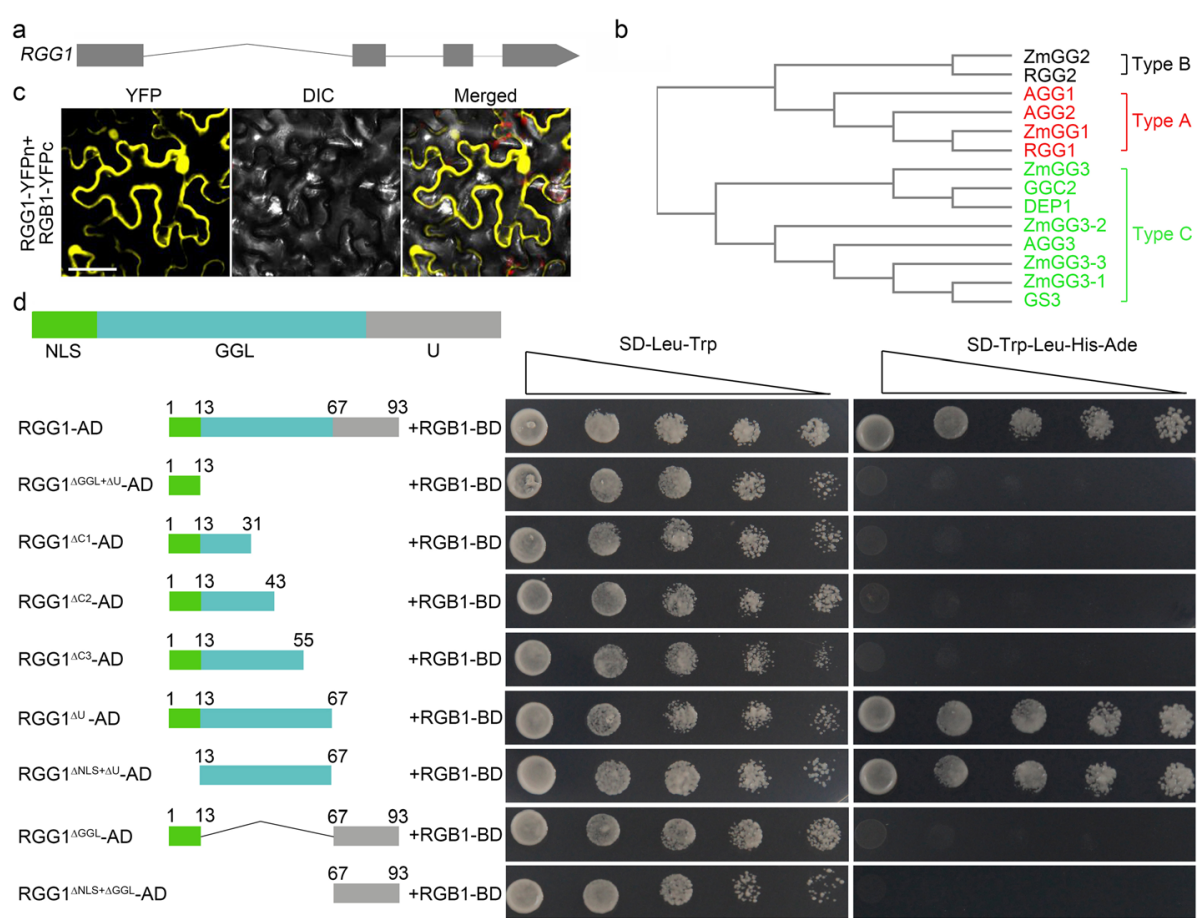

Fig. 1 RGG1 encodes a type-A Gy subunit. a Gene structure of RGG1. b Phylogenetic tree of Gy subunits from rice, Arabidopsis and maize. $\mathbf{c}$ Interaction between RGG1 and RGB1. Scale bars, $100 \mu \mathrm{m}$. $\mathbf{d}$ Yeast two-hybrid assay. In this assay, RGG1 was used as the prey (GAL4-AD, AD) due to its autoactivation ability, and RGB1 was used as the bait (GAL4-BD, BD). $\Delta$ represents deleted protein parts. The numbers show the different lengths of each truncated protein. NLS is the predicted nuclear localization signal. GGL is the G gamma-like domain. $U$ represents the unknown domain

(Fig. 2i). These data suggest that RGG1 may play an important role in panicle and seed development.

To observe the subcellular localization of RGG1, both green fluorescent protein (GFP) and an RGG1-GFP fusion protein driven by the CaMV $35 \mathrm{~S}$ promoter were transiently expressed in rice protoplasts. Similar to the GFP signal, RGG1-GFP was detected in the plasma membrane, cytoplasm and nucleus (Fig. 2j). To verify the function of the predicted NLS at the N-terminus, we also transiently expressed a truncated protein, $R G G 1_{\triangle N L S}-G F P$, in rice protoplasts. However, the fluorescent signal of $R G G 1_{\Delta N L S}-G F P$ showed the same distribution as that of RGG1-GFP, suggesting that the putative NLS domain may not be functional (Fig. S2).

\section{Overexpression of RGG1 Resulted in Yield Reduction in Nipponbare Rice}

To elucidate the biological function of RGG1, overexpression and knockout vectors were generated and then transformed into NIP using an Agrobacterium tumefaciens-mediated method. Several successful transformed lines were obtained and confirmed by qPCR and sequencing. We chose two overexpression (OE) and two mutant lines for further analysis (Fig. 3a, b).

The relative expression levels of RGG1 in two OE lines (OE1 and OE2) were detected. Compared to that in NIP, the expression level of RGG1 was higher by eight- and twelve-fold in OE1 and OE2, respectively (Fig. 3b). As a result, the OE1 and OE2 transgenic lines showed a semidwarf phenotype at maturity (Fig. 3c). Further analysis showed that all the internode lengths of the OE lines were shorter than those of NIP (Fig. S3a, b). Additionally, we quantified other yield components, such as panicle length (PL), tiller number per plant (TN), GN and 1000-grain weight (TGW) (Fig. 3h, i, l, Table S1). Neither PN nor TN showed a difference between NIP and the two OE lines (Fig. 3h, i). However, the TGW values of OE1 and OE2 decreased by $19.20 \%$ and $19.44 \%$, respectively, compared to that of NIP (Fig. 3l). Further analysis suggested that RGG1 affects grain length and width but has no influence on grain thickness (Fig. 3e-k, Table S1). In particular, the grain lengths in the OE lines were lower by $7.41 \%$ and $10.17 \%$, respectively, than that in NIP (Fig. 3j). As expected, OE1 and OE2 also exhibited decreased grain yield per plant (Fig. 3m). Taken together, these results indicate that overexpression of RGG1 can cause semi-dwarf height and shortened grain length.

Additionally, two knockout mutants of RGG1 were generated using the CRISPR/Cas9 system in the NIP background (Fig. 3a). Sequencing results showed that both mutants, NIP-rgg1-1 and NIP-rgg1-2, had large 


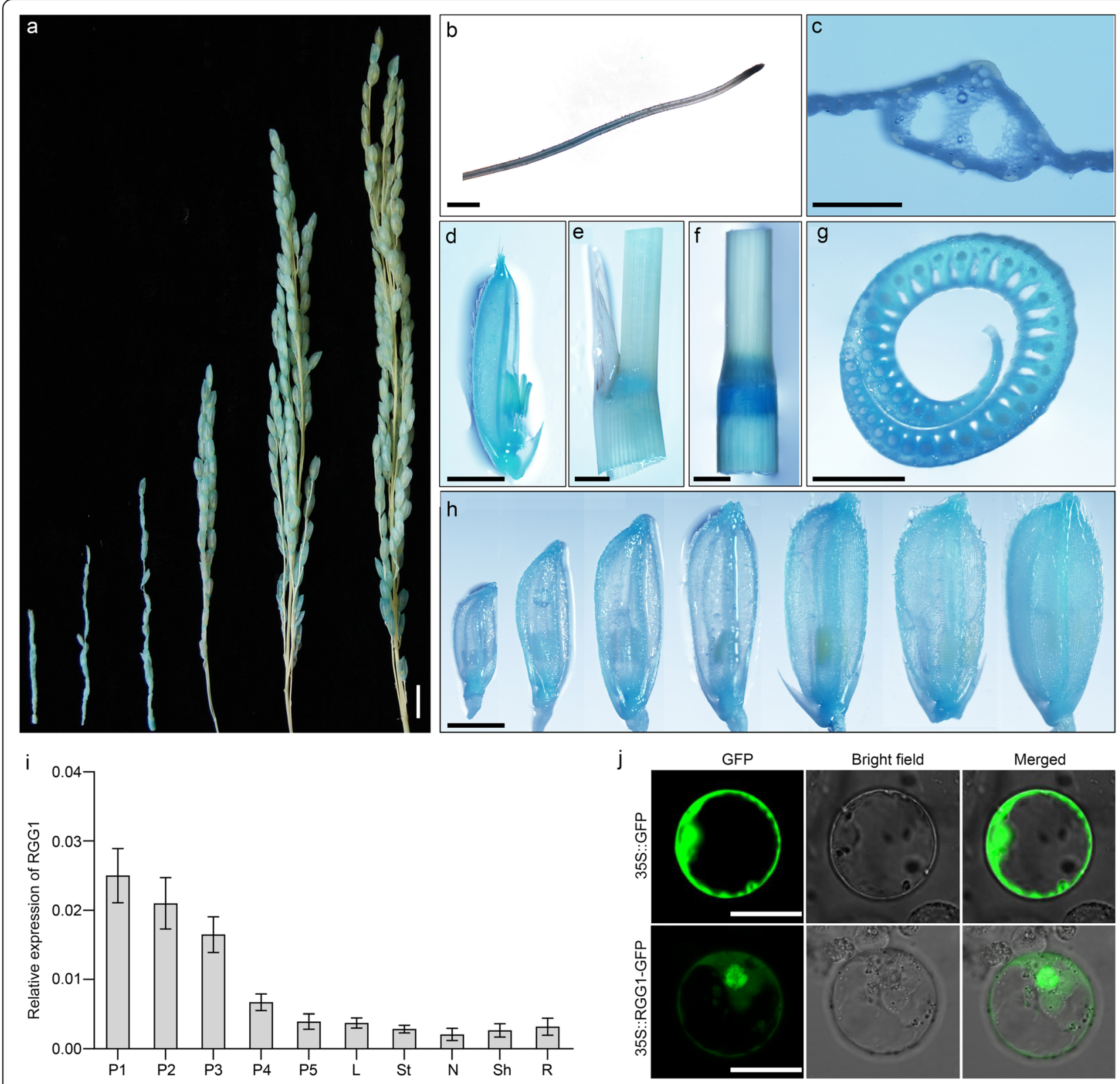

Fig. 2 Molecular characterization of RGG1. a GUS activity in young panicles at different developmental stages. Scale bar, $1 \mathrm{~cm}$. b GUS activity in roots. Scale bar, $1 \mathrm{~mm}$. c GUS activity in leaves. Scale bar, 50 m. d GUS activity in spikelets. Scale bar, $2 \mathrm{~mm}$. e GUS activity in sheaths. Scale bar, $2 \mathrm{~mm}$. $\mathbf{f}$ GUS activity in stem nodes. Scale bar, $2 \mathrm{~mm}$. g GUS activity in node cross-sections. Scale bar, $2 \mathrm{~mm}$. $\mathbf{h}$ GUS activity in spikelets during different developmental stages. Scale bar, $2 \mathrm{~mm}$. i RGG1 transcript levels in different tissues. P1-P5, young panicles with average lengths of approximately $3 \mathrm{~cm}, 6 \mathrm{~cm}, 10 \mathrm{~cm}, 13 \mathrm{~cm}$ and $>15 \mathrm{~cm}$, respectively. L, leaf. St, stem. N, node. Sh, sheath. R, root. j Subcellular localization of RGG1 in rice protoplasts. Scale bar, $20 \mu \mathrm{m}$

deletions in the target site that abolished protein expression. However, the mutant plants of lines NIP-rgg1-1 and NIP-rgg1-2 did not show any obvious phenotype in traits including plant height and other yield component (Fig. 3c-m). This result may be due to the extremely low expression level of RGG1 in NIP (Fig. 3b). Whether the role of $R G G 1$ in signal transduction is subject to genetic redundancy needs further study.

\section{Overexpression of RGG1 in Wunyunjing 30 Results in a Similar Phenotype}

To investigate whether RGG1 shows similar effects to those in the NIP mutants in the qpe9-1/dep1 mutant background, we transformed Wunyunjing 30 (WYJ30), a high-yield variety of rice that naturally lacks a functional qpe9-1/dep1, with the RGG1 overexpression vector, and we measured plant height and other yield-related traits 


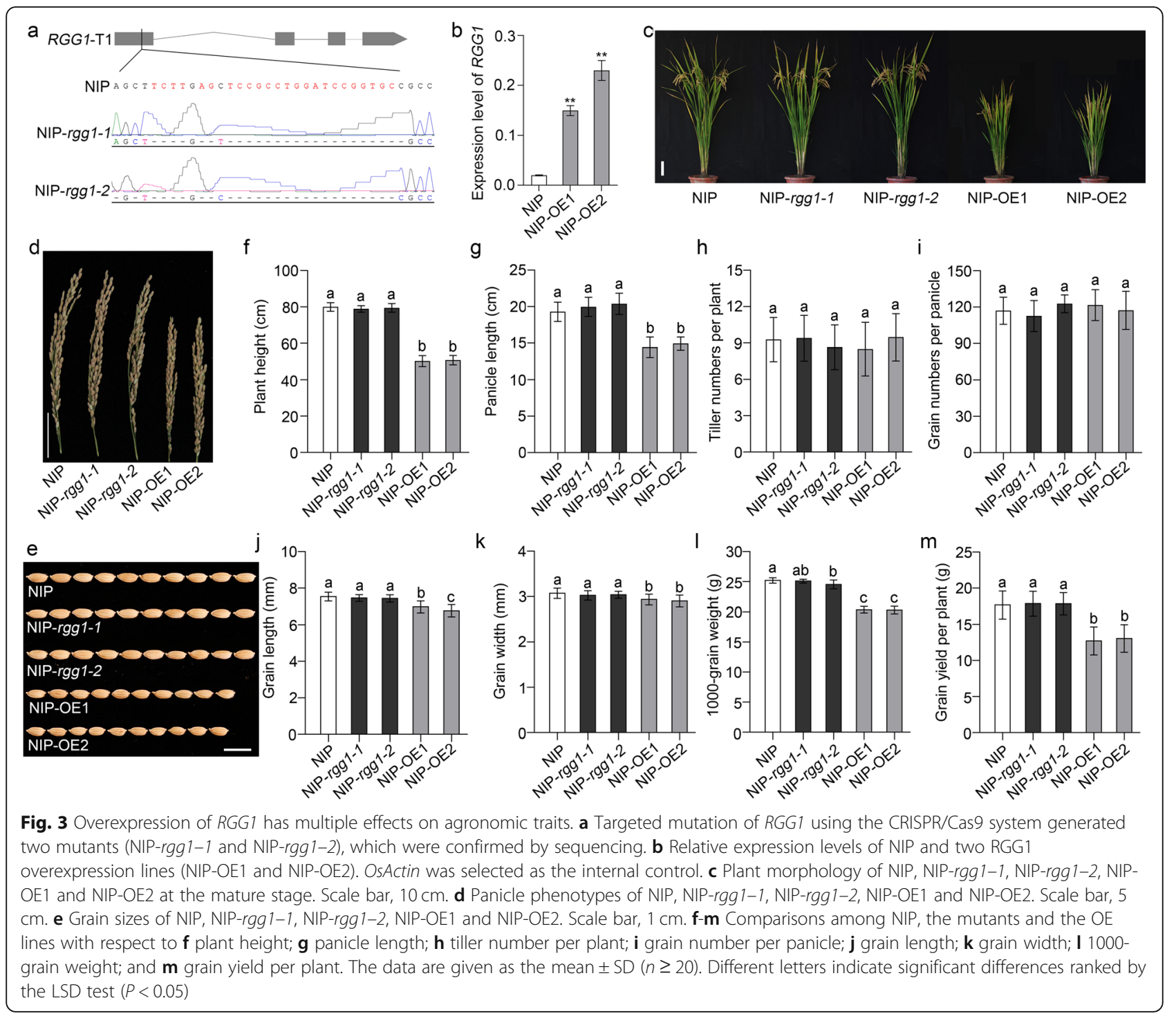

at maturity. Both the WYJ30-OE1 and WYJ30-OE2 lines showed reduced plant height and PL compared to those of WT-WYJ30 (Fig. S4a-d, Table S2). Additionally, compared to WT-WYJ30, the grain lengths of the two OE lines were reduced by $3.29 \%$ and $3.42 \%$, respectively (Fig. S4e). There was no significant difference in grain width between the WYJ30 and OE lines (Fig. S4f). In contrast, overexpressing RGG1 caused decreased TGW and grain yield in WYJ30 (Fig. S4g, h). These results suggest that the roles of $R G G 1$ in regulating plant height and grain length are independent of qPE9-1/DEP1. Pyramiding different $\mathrm{G} \gamma$-encoding genes may be a suitable way to modulate grain size in rice.

We also used the CRISPR/Cas9 method in WYJ30 to obtain several homozygous mutants of RGG1. We identified one line, WYJ30-rgg1-1, with a 4-bp deletion and another with a 1-bp insertion, WYJ30-rgg1-2 (Fig. S5). Both mutations disrupted the GGL domain (Fig. S5). We observed no changes in plant morphology or grain size between WT-WYJ30 and these two mutants (Table S2). Taken together, knockout of RGG1 might not affect rice growth and development.

\section{RGG1 Regulates Grain Size by Affecting Cell Division}

The spikelet hull has an important impact on grain size determination. Compared with WYJ30, both OE lines had reduced grain lengths and grain widths (Fig. 4a, b). Generally, organ size is determined by cell expansion and division. To investigate the grain size differences between the WYJ30 and OE lines, histological crosssections of the spikelet hulls were analysed (Fig. 4c-e). As shown in Fig. 4d and e, both the OE lines had significantly higher cell areas and lower cell numbers than WYJ30. Furthermore, the epidermal cells of WYJ30 and the transgenic lines were analysed using scanning electron microscopy (SEM) (Fig. 4f). No obvious difference 


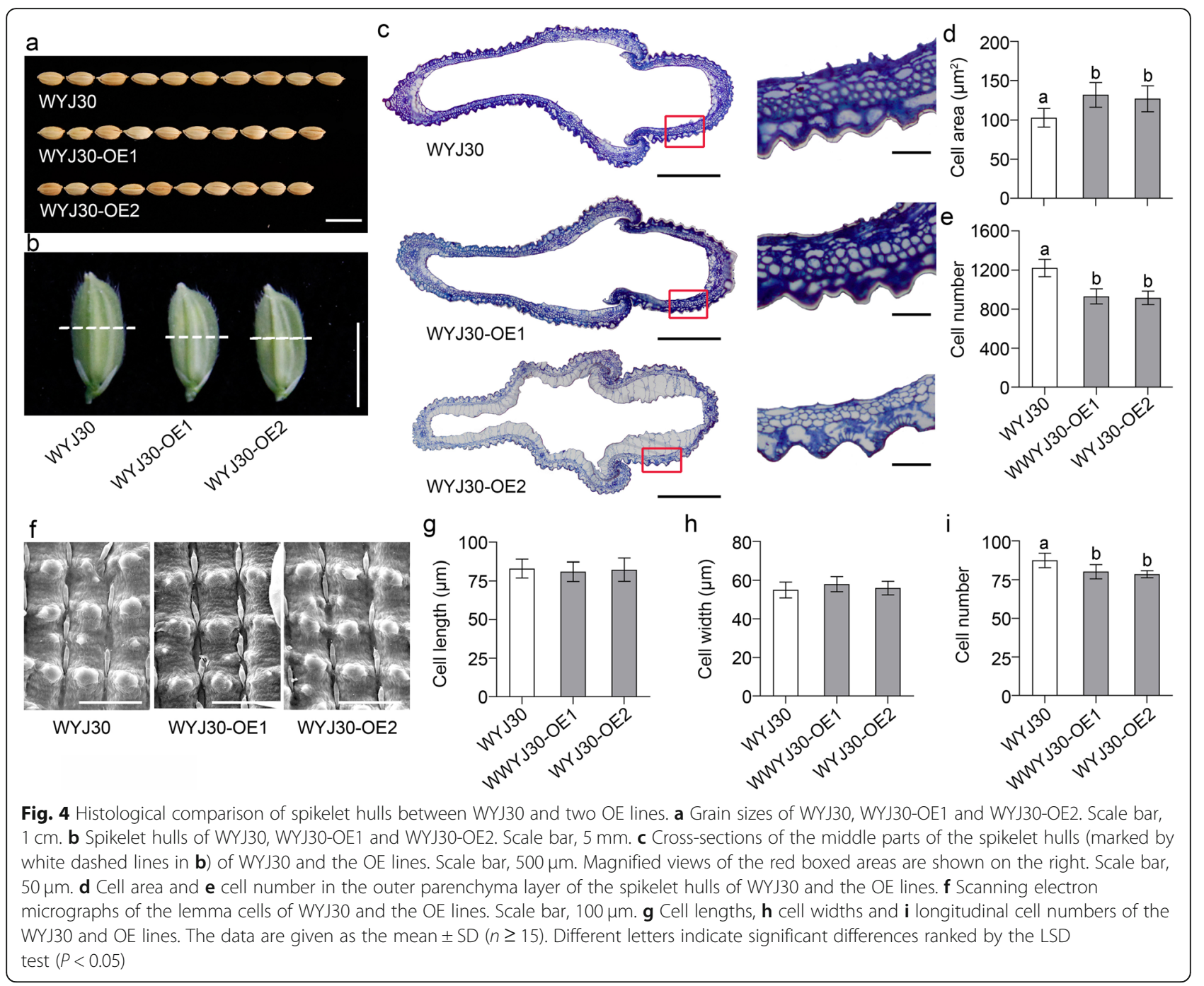

in cell length or cell width was found between the WYJ30 and OE lines (Fig. 4g, h). However, the OE lines had fewer longitudinal cells than WYJ30 (Fig. 4i). Overall, these results suggest that overexpressing RGG1 suppressed cell division in the spikelet hull and consequently led to smaller grain size.

\section{RGG1 is Involved in Cytokinin Biosynthesis}

Due to the significant influences of RGG1 on panicle elongation and grain length that we observed, we then performed a transcriptome analysis to investigate the possible molecular pathway of $R G G 1$ action in the young panicles of NIP, NIP-rgg1-2, and NIP-OE2. A total of 1463 differentially expressed genes (DEGs) were detected in OE2 compared with NIP; 690 of these genes were upregulated, and 773 genes were downregulated (Fig. 5a). Additionally, 754 DEGs, including 249 up- and 505 downregulated genes, were found in the young panicles of the rggl-2 mutant. The detected DEGs were involved in diverse biological processes and metabolic pathways (Fig. S6). Analysis of the DEGs using Gene Ontology showed that the greatest enrichment was in the biological process category. Additionally, Kyoto Encyclopedia of Genes and Genomes (KEGG) analyses revealed that the zeatin biosynthetic pathway was enriched in DEGs (Fig. 5b). In particular, many DEGs were associated with cytokinin biosynthesis (Fig. 5c). Notably, one gene, LOC_ Os01g40630, encoding the cytokinin-activating enzyme LOG, which is responsible for converting inactive cytokinin to biologically active forms, was downregulated in the young panicles of NIP-OE2 (Fig. 5c, d). The expression levels of several cytokinin biosynthetic genes were confirmed using qPCR assays (Fig. S7a-c), and LOG was found to be downregulated in the young panicles of the OE lines and upregulated in rgg1 mutants. We also analysed two other cytokinin biosynthetic genes. CYP735A4, encoding the key enzyme converting isopentenyladenine (iP)-type to trans-zeatin (tZ)-type cytokinins, had 


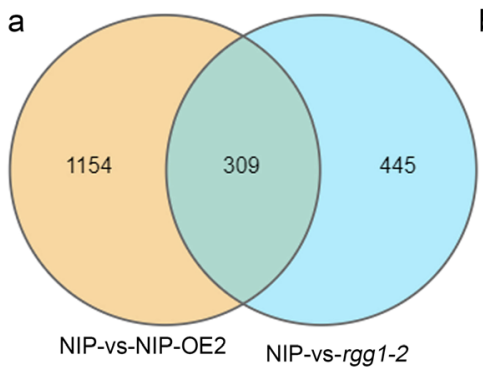

C

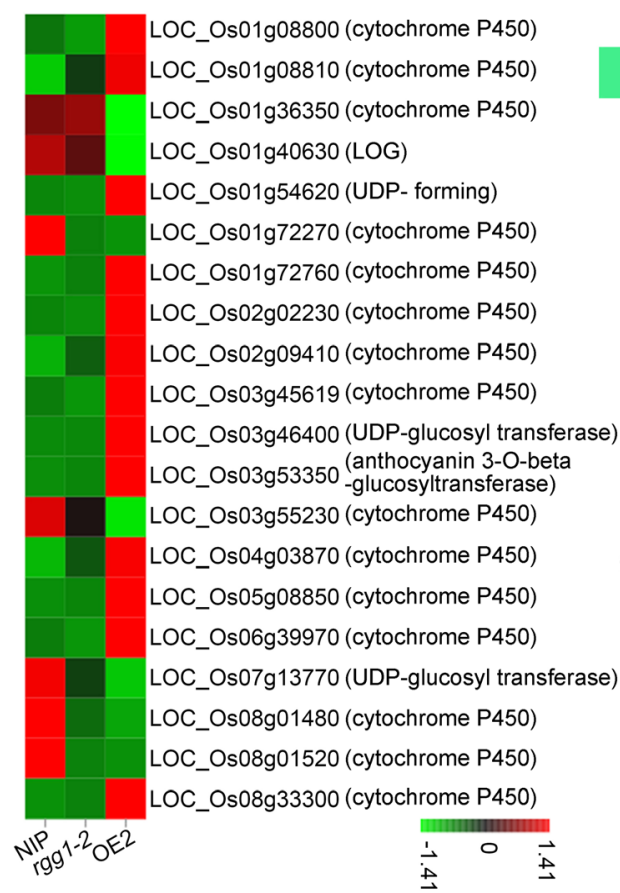

\section{b}

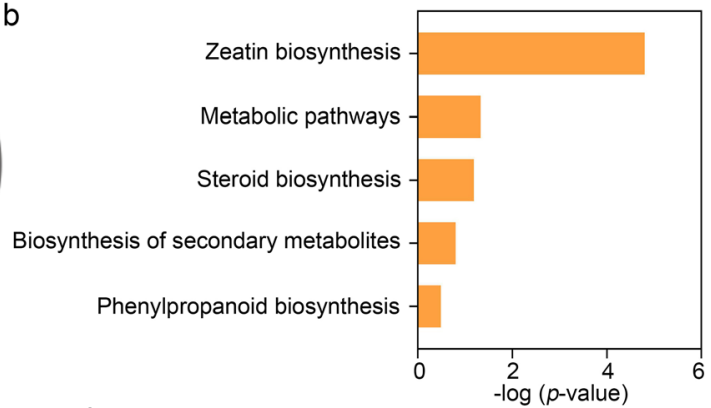

d

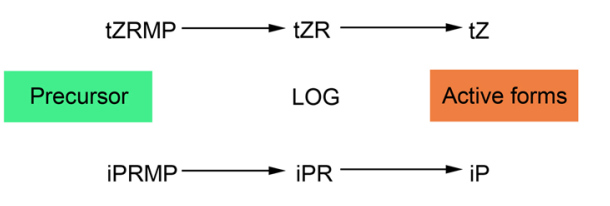

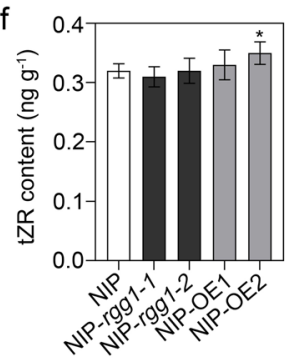
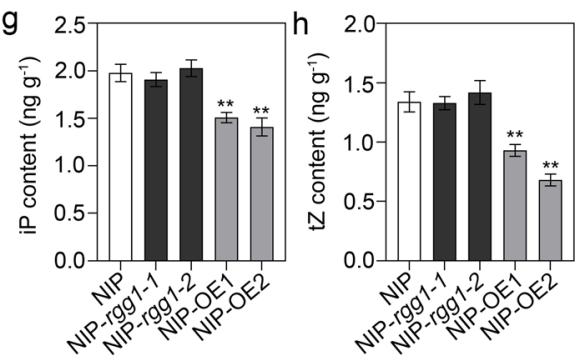

Fig. 5 Transcriptome profiles of NIP, rgg1-2 and NIP-OE2. a Differentially expressed genes (DEGs) in the young panicles of NIP and the transgenic lines. The young panicles of NIP, rgg1-2 and NIP-OE2, including 3 biological replicates, were collected for analysis. b Kyoto Encyclopedia of Genes and Genomes (KEGG) enrichment of DEGs. $\mathbf{c}$ Heat map of DEGs associated with cytokinin biosynthetic and regulatory pathways. The different colours in each box indicate the Z-score values. $\mathbf{d}$ Schematic of the cytokinin biosynthetic pathway from precursors (tZRMP and iPRMP) to active forms (tZ and iP). tZRMP, trans-zeatin riboside-5'-monophosphate. iPRMP, N6-( $\Delta$ 2-isopentenyl) adenine riboside monophosphate. tZ, trans-zeatin. $\mathrm{iP}$, isopentenyladenine. e iPR content, $\mathbf{f}$ tZR content, $\mathbf{g} \mathrm{iP}$ content and $\mathbf{h}$ tZ content in young panicles of NIP and the transgenic lines (with three biological replicates). iPR, N6-( $\Delta$ 2-isopentenyl)adenosine. tZR, trans-zeatin-riboside. The data are given as the mean \pm SD $(n=3)$. Student's $t$-test: ${ }^{*} P<0.05,{ }^{* *} P<0.01$

decreased expression in the panicles of the OE lines, while no significant changes were observed in the mutants. OsIPT9 encodes IPP transferase for synthesizing $\mathrm{cZ}$ in rice (Tsai et al. 2012) and was downregulated in the young panicles of the OE lines and upregulated in the rgg1 mutants. These results suggest that RGG1 might be involved in the cytokinin regulatory pathway.

To test this hypothesis, we measured the concentrations of cytokinin in young panicles (Fig. 5e-h). The total contents of two cytokinin precursors, N6-( $\Delta 2$-isopentenyl) adenosine (iPR) and trans-zeatin-riboside (tZR), in the OE lines were similar to those in NIP (Fig. 5e, f). However, the contents of the active forms, iP and $\mathrm{tZ}$, were significantly lower in the OE lines than in NIP (Fig. 5g, h). NIP-OE2 accumulated more tZR than NIP did, and this effect may be due to an inefficient conversion ability (Fig. 5f). These results suggest that overexpression of RGG1 reduced the efficiency of the conversion of cytokinin precursors to active forms, possibly as a result of lower expression of $L O G$ or other genes in the cytokinin pathway.

\section{RGG1 Affects Cytokinin Signalling}

Heterotrimeric GTP binding proteins ( $G$ proteins) are involved in multiple signal transduction processes and intracellular responses to stimuli in plants. We also 
investigated whether RGG1 affects cytokinin signal transduction in rice. Shoot and root elongation assays were conducted to test the sensitivity of the overexpression and mutant lines to different concentrations of 6benzylaminopurine (6-BA) (Fig. 6a). These experiments revealed an altered growth curve for the RGG1 overexpression lines when treated with 6-BA. At low concentrations, the shoot elongation of NIP and the two mutants was more strongly inhibited than that of OE1 and OE2 (Fig. 6b). The inhibition of root elongation by cytokinin was also compared between NIP and transgenic lines (Fig. 6c). These results showed that the two OE lines had reduced sensitivity to 6-BA with respect to its inhibitory effect on root elongation (Fig. 6c). All these results indicated that $R G G 1$ is involved in cytokinin biosynthesis and signal transduction in rice.

\section{Discussion}

Grain size is one of the important agronomic traits affecting rice yield and quality. Although a complex regulatory gene network related to grain size has been proposed (Miao et al. 2019; Li and Li 2016), the crosstalk between heterotrimeric $\mathrm{G}$ proteins and the cytokinin regulatory pathway in grain size control is poorly understood. In this study, we show that overexpression of RGG1 resulted in decreased plant height and grain length (Fig. 2 and Fig. S4). Physiological measurements suggested that the active cytokinin level was lower in OE lines than in NIP (Fig. 5), and a 6-BA treatment assay showed that RGG1 was involved in cytokinin signal transduction. Thus, our findings demonstrate that RGG1 is involved in cytokinin biosynthesis and signalling and thus controls grain size as well as plant height.

Phylogenetic analysis revealed that rice RGG1 is a type-A Gy protein. It contains a conserved GGL domain and a CaaX motif at the C-terminus that is characteristic of $\mathrm{G} \gamma$ subunits among plants and animals and ensures proper membrane targeting (Pandey 2019). Therefore, RGG1 represents a canonical Gy protein. Subcellular localization results showed that RGG1 localizes to the plasma membrane, cytoplasm, and nucleus (Fig. 2j). Generally, $G \alpha$ and $G \beta \gamma$ dimers perceive a stimulus at the surface of the cell when in contact with the membrane and then separate to transmit the signal to downstream effectors (Hildebrandt et al. 1984). Previous studies have shown that RGB1, RGG1 and RGG2 localize to the plasma membrane (Kato et al. 2004). However, it is difficult to understand the roles of $G$ proteins within the nucleus. In the nuclei of mammalian cells, $G \beta \gamma$ heterodimers interact with a transcription factor, AP-1, and thus are likely have a transcriptional regulatory role there (Chang et al. 2013; Robitaille et al. 2010). In rice, GS3GFP and DEP1-GFP are detectable in the membrane and nucleus and function as cofactors of OsMADS1 in regulating grain size (Liu et al. 2018). More recently, the type B G $\beta$ subunit RGG2 was also found to be localized

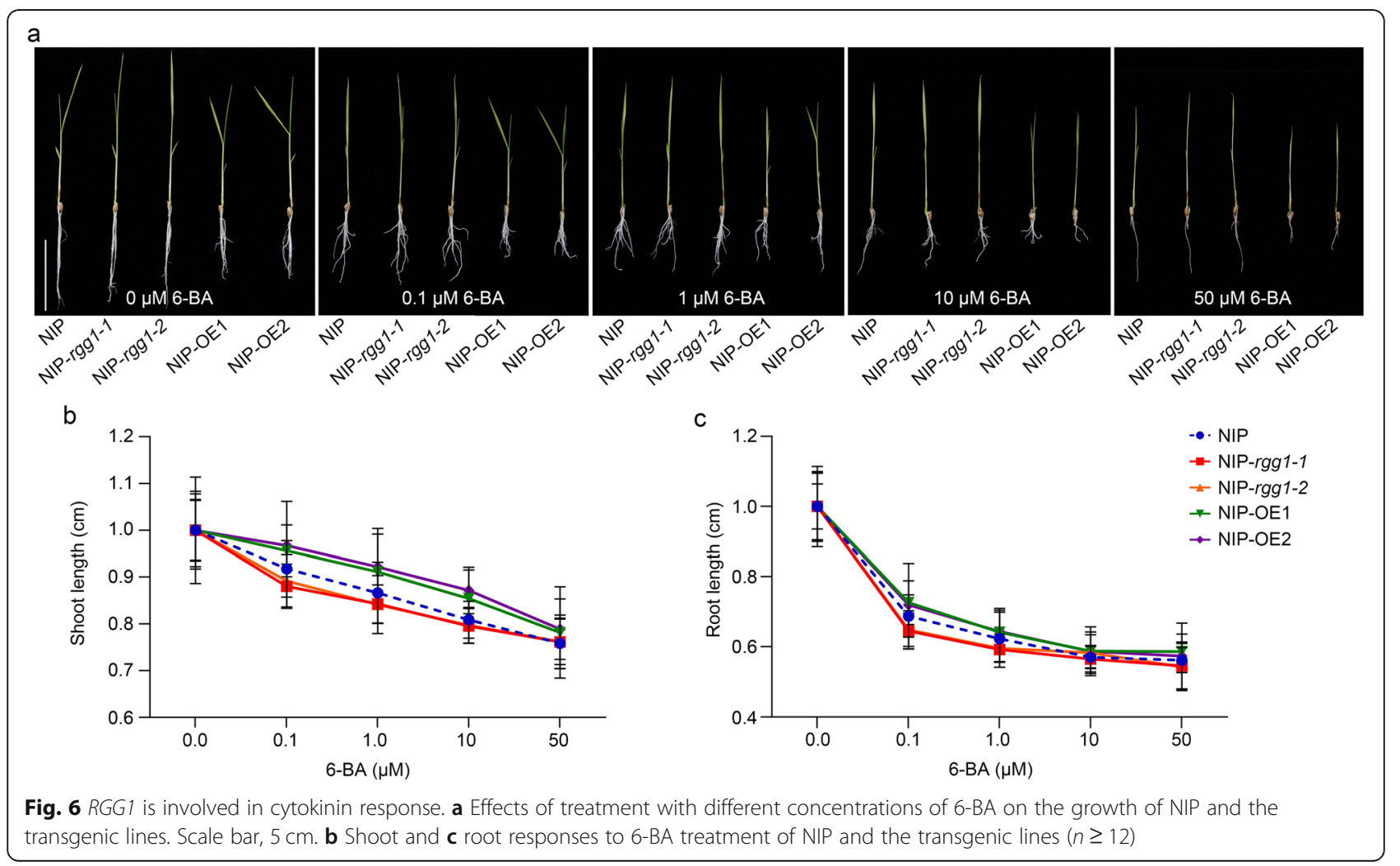


to the plasma membrane, cytoplasm and nucleus (Miao et al. 2019). All of these results suggest that localization of $G$ proteins, including RGG1, to the nucleus is associated with $G$ protein signalling and functions in transcriptional regulation in rice.

Our qPCR and GUS staining results showed that RGG1 was constitutively expressed in diverse tissues, especially in panicles and spikelets (Fig. 2a-i). To investigate the roles that $R G G 1$ plays in panicle development, we successfully generated RGG1 knockout and overexpression lines. The knockout lines $\operatorname{rgg} 1-1$ and $r g g 1-2$ had no obviously different phenotypes when compared to NIP (Fig. 3, Tables S1 and S2). One possible reason is that RGG1 normally has very low expression levels in rice (Fig. 3b). In Arabidopsis, the agg1 and agg2 mutants and agglagg2 double mutant exhibit no changes in rosette size, while triple agglagg2agg3 mutants show a reduction in rosette size (Thung et al. 2012). Therefore, another possible explanation is that $R G G 1$ has a redundant function with respect to signal transduction. In contrast, the overexpression lines OE1 and OE2 showed decreased plant height and PL as well as small grains, all of which are consistent with results from a previous study (Liu et al. 2018). In addition, Swain et al. reported that increased RGG1 expression resulted in increased plant height and enhanced tolerance to salinity stress (Swain et al. 2017). Therefore, we evaluated salinity stress tolerance using our transgenic lines in the NIP background. However, seedlings from the $\mathrm{OE}$ lines did not show increased tolerance to salinity stress under treatment with $200 \mathrm{mM} \mathrm{NaCl}$ (Fig. S8). Thus, our results support yield reduction and reduced plant height when $R G G 1$ is overexpressed but not increased height or salt tolerance.

We also overexpressed RGG1 in the WYJ30 background, which contains a loss-of-function allele of qpe91/dep1. Similar phenotypes, including a semi-dwarf plant architecture, shortened PL, decreased grain length, and overall lower yield, were also observed in the $\mathrm{OE}$ lines (Fig. S4, Table S2). Therefore, RGG1 and $q P E 9-1 / D E P 1$ may function differently in controlling grain size and grain yield characteristics, especially given that WYJ30 is a high-yield variety. Although the roles of $G \beta$ and $G \gamma$ subunits in regulating grain size are well known, the mechanisms by which $G$ proteins mediate this process remain poorly understood. For example, Sun et al. crossed GS3-1Ri with RGB1Ri transgenic plants and found that the RGB1Ri/GS3-1Ri hybrid showed reduced grain length. This finding suggests that the effects of grain length increase by GS3-1Ri were dependent on RGB1. Similar results were also obtained in the RGB1Ri/ DEP1OE and RGB1Ri/GGC2OE plants. Therefore, GS3 may have no effect on regulating grain size by itself, while DEP1 and GGC2 compete with RGB1 to modulate grain size. Additionally, a previous study showed that $\mathrm{G}$ protein $\beta$ and $\gamma$ subunits could physically interact with the transcription factor OsMADS1 to promote its transcriptional activity, thereby controlling grain morphology (Liu et al. 2018). Recently, we functionally analysed one gene, $R G G 2$, and found that $R G G 2$ negatively regulates grain size via the gibberellin pathway (Miao et al. 2019). These prior studies and our present results suggest that the mechanism of regulation of grain size by $G$ proteins is likely very complicated. However, a more complete understanding of how $G$ proteins operate to control grain size in rice is urgently needed to better manipulate rice grain size to meet global consumer demands.

To understand the possible regulatory pathway that RGG1 mediates, we performed a transcriptome analysis using young panicles from NIP and transgenic plants. The results showed that many DEGs were associated with cytokinin biosynthesis (Fig. 5). In particular, one gene, $L O G$, showed significantly lower expression in the OE lines (Fig. 5c and Fig. S7a). LOG is responsible for converting cytokinin precursors to bioactive forms, and its mutant has a defect in inflorescence meristem development (Kurakawa et al. 2007). Cytokinins are adenine derivatives that play essential roles in regulating shoot meristem development (Hwang et al. 2012). However, the relationship between cytokinins and $\mathrm{G}$ proteins in mediating developmental processes in plants is poorly understood. In Arabidopsis, in addition to the canonical $\mathrm{G} \alpha$ protein, there are three EXTRA-LARGE G $\alpha$-like PROTEINs (XLGs) that interact with U-box, PUB, E3 ligases, PUB2 and PUB4, and both the triple mutant xlgs and the double mutant $p u b 2 / 4$ showed defects in cytokinin response. Within the mutant lines, overexpression of $A R R 10$, a positive cytokinin response regulator, partially rescued the defective phenotypes (Wang et al. 2017). Recently, Zhang et al. reported that $q P E 9-1 / D E P 1$ positively regulates grain filling by increasing auxin and cytokinin content in rice grains. Here, we revealed that the concentration of endogenous cytokinin was decreased in the OE lines compared with that in NIP due to the decreased expression of genes encoding cytokinin biosynthetic enzymes. Cytokinin signalling comprises a classic two-step phosphorelay system where an initial signal is transferred to a response regulator (Argueso et al. 2010; Hwang et al. 2012). Here, we also found that $R G G 1$ is involved in cytokinin signal transduction based on a 6-BA treatment assay (Fig. 6a-c).

Overall, our findings, along with prior work, help to show the complicated crosstalk between $G$ proteins and cytokinin. The critical nature of $G$ proteins in plant development is highlighted by recent reports of knockouts of $\mathrm{G} \beta(R G B 1)$ or $\mathrm{G} \gamma(R G G 2)$ in rice or $\mathrm{G} \beta$ $(\mathrm{ZmGB1})$ in maize causing lethal phenotypes (Gao et al. 2019; Miao et al. 2019; Wu et al. 2020). This may 
be unsurprising given that $\mathrm{G}$ proteins probably mediate shoot meristem size through interaction with CLAVATA receptors (Bommert et al. 2013; Ishida et al. 2014; Wu et al. 2020) and may impact embryo formation via a network between $G$ proteins and cytokinin. We expect that further elucidating the interplay between cytokinin and $\mathrm{G}$ proteins will be beneficial for crop improvement via genetic engineering and molecular breeding.

\section{Conclusions}

Altogether, our results showed that overexpression of RGG1 significantly decreased plant height, panicle length and grain length by regulating cell division in rice. Furthermore, our findings suggested that RGG1 is involved in cytokinin biosynthesis and signalling pathway. Thus, this study reveals a novel $G$ protein-cytokinin module controlling grain size in rice and will be beneficial for understanding the mechanisms by which $G$ proteins regulate grain size and plant development.

\section{Materials and Methods}

\section{Plant Materials and Growth Conditions}

Both wild-type cultivars (NIP and WYJ30) and transgenic lines of the $T_{3}$ generation were used for phenotypic analyses. These materials were grown on the experimental farm of Yangzhou University following normal agricultural practices. For analyses at the seedling stage, plants were grown in hydroponic culture in a growth chamber with a 12 -h light $\left(30^{\circ} \mathrm{C}\right)$ and 12 -h dark $\left(28^{\circ} \mathrm{C}\right)$ photoperiod and $70 \%$ humidity.

\section{Homologous Detection and Phylogenetic Analysis}

The sequences of the rice, Arabidopsis and maize $\mathrm{Gr}$ proteins were obtained from NCBI (https://www.ncbi. nlm.nih.gov/). Multiple alignments were performed using Clustal X. Maximum likelihood (ML) and neighbour-joining (NJ) methods were adopted for the phylogenetic analysis using MEGA v7.0. The ML phylogenetic analyses were conducted with the following parameters: Jones-Taylor-Thornton (JTT) model, estimated proportion of invariable sites, 4 rate categories, estimated gamma distribution parameter, and optimized starting BIONJ tree. In addition, the JTT model was employed for the construction of $\mathrm{NJ}$ trees. A total of 1000 non-parametric bootstrap samplings were carried out to estimate the support level for each internal branch for both the ML and NJ trees.

\section{Vector Construction and Rice Transformation}

To construct the RGG1-OE vector, the full-length coding sequence was amplified from NIP cDNA and then inserted into the $\mathrm{p} 1301 \mathrm{Ubi}$ vector. To generate $\mathrm{pC} 1300$ Cas9-g ${ }^{R G G 1}$ mutants, we designed a target sequence in the first exon, and the final fragment was inserted into the $\mathrm{pC} 1300-\mathrm{Cas} 9$ vector. The $2.0-\mathrm{kb}$ promoter sequence of $R G G 1$ was cloned to drive the $\beta$-glucuronidase (GUS) gene, and the promoter-GUS vector was transformed into NIP. All these vectors were introduced into Agrobacterium tumefaciens strain EHA105 for subsequent transformation of NIP or WYJ30. Homozygous $\mathrm{T}_{2}$ generation plants were used for further analysis.

All primers used in this study are listed in Supplemental Table S3.

\section{Histochemical GUS Staining and Subcellular Localization Analysis}

For GUS staining, positive transgenic plants were selected at different developmental stages using an X-Gluc kit (Real-Times (Beijing) Biotechnology Co. Ltd.). Leaves, nodes, sheaths, stems, roots, and young panicles of different stages were collected and then soaked in solution with X-Gluc in a $37^{\circ} \mathrm{C}$ dark environment for one night. Then, samples were cleared with absolute ethanol for observation.

For subcellular localization analysis, the full-length cDNA of the RGG1 gene was amplified and cloned into the pCAMBIA1300-221GFP vector to generate 35S: RGG1-GFP. The construct was directly transformed into rice protoplasts, and the GFP signals were observed by confocal microscopy (Leica). The primers used are listed in Supplemental Table S3.

\section{Evaluation of Agronomic Traits}

Before harvest, several yield-related agronomic traits were measured, including plant height, internode length, $\mathrm{TN}$, and PL of the main stem. Grain-related traits, including grain length, grain width, and TGW, were measured after harvesting and stored at $37^{\circ} \mathrm{C}$ for 1 week. The total seeds of one plant, minus any empty grains, were weighed to determine the grain yield per plant. Data statistics and sample $t$-tests were analysed using Excel (2016) software.

\section{Histological Analysis}

Fresh young spikelet hulls of WYJ30 and the WYJ30-OE lines were collected, fixed in $2.5 \%$ glutaraldehyde for more than $24 \mathrm{~h}$ and then dehydrated through a graded alcohol-isoamyl acetate series. Images of cross-sections were taken on a Zeiss Axioskop HBO 50 or a Leica MZFLIII fluorescence stereomicroscope. For glume cell observation, the outer surfaces of mature seeds were observed by SEM (S-4800, Hitachi). The cell number and cell area in the outer parenchyma cell layer were measured using ImageJ software. 


\section{RNA Extraction and qPCR}

Total RNA was extracted using an RNA extraction kit (Beijing Tiangen Biotechnology Co. Ltd.). High-quality RNA was used to generate cDNA using a FastQuant RT Kit (Beijing Tiangen Biotechnology Co. Ltd.). Gene expression levels were analysed using qPCR. The rice Actin gene was used as an internal control. The qPCR was carried out in a total volume of $20 \mu \mathrm{L}$, containing $2 \mu \mathrm{L}$ of cDNA, $10 \mu \mathrm{M}$ of each primer, $10 \mu \mathrm{L}$ of $2 \times$ SYBR Green PCR Master Mix, and $0.4 \mu \mathrm{L}$ of $50 \times$ ROX Reference Dye 2 (Vazyme Biotech Co. Ltd.), and performed on an ABI ViiA 7 Real-Time PCR System. The primers used for qPCR are listed in Supplemental Table S3.

\section{Cytokinin Measurement and Treatment}

For measurement of cytokinin, young panicles of NIP and transgenic plants were collected in liquid nitrogen. Cytokinin measurement was performed as previously described (Cai et al. 2014). For cytokinin treatment, one-week-old seedlings were grown in hydroponic medium and then treated with different concentrations of 6-BA. After 1 week of treatment, the shoot and root length were measured to analyse the response to cytokinin. For gene expression analysis, 10-d-old seedlings were grown in hydroponic medium containing $10 \mu \mathrm{M}$ 6-BA. Leaves were collected every $2 \mathrm{~h}$ for RNA extraction, and the expression level of OsRR9 was detected by qPCR.

\section{RNA-Sequencing Analysis}

Young panicles of NIP and the transgenic lines were collected for total RNA extraction using a TRIzol reagent kit (Invitrogen, Carlsbad, CA, USA). Construction of the cDNA library and sequencing were performed at Gene Denovo Biotechnology Co. (Guangzhou, China) using the Illumina HiSeq 2500 platform (Illumina Inc., San Diego, CA, USA). The filtered clean reads were aligned to the rice NIP reference genome and genes (http://rice.plantbiology.msu. edu/) using HISAT2. 2.4. RNA differential expression analysis was performed by DESeq2.

\section{Yeast Two-Hybrid Assay}

To detect the interactions between RGG1 and RGB1, the full-length and truncated sequences of RGG1 were cloned into the pGADT7 vector, and RGB1 was cloned into the pGBKT7 vector. Yeast two-hybrid assays were performed according to the manufacturer's user manual. The primers listed in Supplemental Table S3.

\section{BiFC Analysis}

For the BiFC assay, the coding sequence of RGG1 was cloned into pCAMBIA1300-35S-N-YFPn, and the coding sequence of RGB1 was cloned into the pCAMBIA1300-35S-
N-YFPc vector. The plasmids were electroporated into $A$. tumefaciens (strain GV3101) and coinfiltrated into tobacco (Nicotiana benthamiana) leaves. After infiltration for 2-3 days, the GFP signals were observed by confocal microscopy (Leica). The primers used for BiFC are listed in Supplemental Table S3.

\section{Statistical Analysis}

The results are presented as the mean \pm SD. Microsoft Excel 2016 was used for statistical testing. GraphPad Prism 8 was used to produce bar charts and line charts. Significance levels were determined according to Student's $t$-test: ${ }^{*} P<0.05,{ }^{* *} P<0.01$.

\section{Supplementary Information}

The online version contains supplementary material available at https://doi. org/10.1186/s12284-020-00436-x

\begin{abstract}
Additional file 1 : Fig. S1. Sequence alignment of the Gy proteins in rice, Arabidopsis and maize. The red line indicates the predicted nuclear localization signal sequence. The red box indicates the CaaX

isoprenylation motif at the C-terminal end. The green line indicates the GGL domain. Fig. S2. Subcellular localization of RGG1 ${ }^{\Delta N L S}$-GFP. Scale bar, $20 \mu \mathrm{m}$. Fig. S3. Internode lengths of NIP and the RGGI OE lines. Scale bar, $5 \mathrm{~cm}$. Fig. S4. Comparison of plant and grain phenotypes between WYJ30 and the OE lines. Fig. S5. Targeted mutagenesis of RGG1 in the WYJ30 background and protein sequence alignment of WYJ30 and the mutants. Fig. S6. Gene Ontology (GO) DEG enrichment of the biological process, molecular function and cellular component categories. Fig. S7. Relative expression levels of genes concerning cytokinin biosynthesis.

Fig. S8. $\mathrm{NaCl}$ treatment of NIP and the transgenic lines. Table S1. Comparison of major agronomic traits between NIP and the transgenic lines. Table S2. Comparison of major agronomic traits between WYJ30 and the transgenic lines. Table S3. Primers used in this study.
\end{abstract}

\section{Abbreviations}

G proteins: Heterotrimeric GTP binding proteins; GUS: $\beta$-glucuronidase; NIP: Nipponbare; WYJ30: Wuyunjing 30; DEGs: Differentially expressed genes; 6-BA: 6-benzylaminopurine; GPCRs: Seven-pass transmembrane G proteincoupled receptors; SAM: Shoot apical meristem; LOG: LONELY GUY; CT2: COMPACT PLANT2; CLV2: FASCIATE EAR2; BG3: Big Grain3; qPE9-1/ DEP1: DENSE AND ERECT PANICLE 1; ren1-D: Root enhancer1;

OsCKX4: Cytokinin oxidase 4; OsPUP4: Purine permease 4; GGL: G gammalike; NLS: Nuclear location signal; BiFC: Bimolecular fluorescent complementation; qPCR: Quantitative reverse transcription-PCR; GFP: Green fluorescent protein; PL: Panicle length; TN: Tiller number per plant; GN: Grain number; TGW: 1000-grain weight; GL: Grain length; GW: Grain width; GYPP: Grain yield per plant; SEM: Scanning electron microscopy; KEGG: Kyoto Encyclopedia of Genes and Genomes; iP: Isopentenyladenine; tZ: Transzeatin; iPR: N6-( $\Delta 2$-isopentenyl) adenosine; tZR: Trans-zeatin-riboside; XLGs: EXTRA-LARGE Ga-like PROTEINs

\section{Acknowledgements}

We would like to thank all our colleagues at the Jiangsu Academy of Agricultural Sciences and Yangzhou University for reading and participating in discussions relating to the preparation of this manuscript.

\section{Authors' Contributions}

YJ, LGH, and ZY designed the experiments. TYJ and MJ wrote this paper and performed most of the experiments with the assistance of $C Z \mathrm{H}$ and JYJ. ZL, WJ, and FFJ performed phenotype measurements. XY, LWQ, and WFQ constructed some of the vectors. TYJ and MJ contributed equally to this work. The author(s) read and approved the final manuscript. 


\section{Funding}

This study was funded by the National Natural Science Foundation of China (31901525), the Natural Science Foundation of Jiangsu Province (BK20190255), the Jiangsu Province Key Research and Development Program (Modern Agriculture) Project (BE2019342-2), and the Jiangsu Agricultural Science and Technology Innovation Fund (CX(18)2022).

\section{Availability of Data and Materials}

The datasets supporting the conclusions of this article are included within the article and its additional files.

\section{Ethics Approval and Consent to Participate}

Not applicable.

\section{Consent for Publication}

Not applicable.

\section{Competing Interests}

The authors declare that there are no conflicts of interest.

\section{Author details}

'Institute of Food Crops, Jiangsu Academy of Agricultural Sciences/Nanjing Branch of Chinese National Center for Rice Improvement, Nanjing 210014, Jiangsu, China. ${ }^{2}$ Jiangsu Co-Innovation Center for Modern Production Technology of Grain Crops, Yangzhou University, Yangzhou 225009, Jiangsu, China. ${ }^{3}$ Jiangsu Key Laboratory of Crop Genetics and Physiology/Key Laboratory of Plant Functional Genomics of the Ministry of Education, Yangzhou University, Yangzhou 225009, China.

\section{Received: 6 July 2020 Accepted: 30 October 2020}

\section{Published online: 10 November 2020}

\section{References}

Argueso CT, Raines T, Kieber JJ (2010) Cytokinin signaling and transcriptional networks. Curr Opin Plant Biol 13:533-539

Ashikari M, Sakakibara H, Lin S, Yamamoto T, Takashi T, Nishimura A, Angeles ER, Qian Q, Kitano H, Matsuoka M (2005) Cytokinin oxidase regulates rice grain production. Science 309:741-745

Blázquez M, Nelson D, Weijers D (2020) Evolution of plant hormone response pathways. Annu Rev Plant Biol. https://doi.org/10.1146/annurev-arplant050718-100309

Bommert P, Je Bl, Goldshmidt A, Jackson D (2013) The maize Galpha gene COMPACT PLANT2 functions in CLAVATA signalling to control shoot meristem size. Nature 502:555-568

Cai BD, Zhu JX, Gao Q, Luo D, Yuan BF, Feng YQ (2014) Rapid and highthroughput determination of endogenous cytokinins in Oryza sativa by bare $\mathrm{Fe}_{3} \mathrm{O}_{4}$ nanoparticles-based magnetic solid-phase extraction. J Chromatogr A 1340:146-150

Chang C, Lee L, Yu D, Dao K, Bossuyt J, Bers D (2013) Acute $\beta$-adrenergic activation triggers nuclear import of histone deacetylase 5 and delays $\mathrm{G}(\mathrm{q})$ induced transcriptional activation. J Biol Chem 288:192-204

Gao S, Fang J, Xu F, Wang W, Sun X, Chu J, Cai B, Feng Y, Chu C (2014) Cytokinin oxidase/dehydrogenase4 integrates cytokinin and auxin signaling to control rice crown root formation. Plant Physiol 165:1035-1046

Gao Y, Gu H, Leburu M, Li X, Wang Y, Sheng J, Fang H, Gu M, Liang G (2019) The heterotrimeric $G$ protein $\beta$ subunit $R G B 1$ is required for seedling formation in rice. Rice 12.53

Hildebrandt JD, Codina J, Risinger R, Birnbaumer L (1984) Identification of a gamma subunit associated with the adenylyl cyclase regulatory proteins Ns and Ni. J Biol Chem 259:2039-2042

Huang X, Qian Q, Liu Z, Sun H, He S, Luo D, Xia G, Chu C, Li J, Fu X (2009) Natural variation at the DEP1 locus enhances grain yield in rice. Nat Genet 41:494-497

Hwang I, Sheen J, Muller B (2012) Cytokinin signaling networks. Annu Rev Plant Biol 63:353-380

Ishida T, Tabata R, Yamada M, Aida M, Mitsumasu K, Fujiwara M, Yamaguchi K, Shigenobu S, Higuchi M, Tsuji H, Shimamoto K, Hasebe M, Fukuda H, Sawa S (2014) Heterotrimeric G proteins control stem cell proliferation through CLAV ATA signaling in Arabidopsis. EMBO Rep 15:1202-1209
Kato C, Mizutani T, Tamaki H, Kumagai H, Kamiya T, Hirobe A, Fujisawa Y, Kato H, Iwasaki Y (2004) Characterization of heterotrimeric G protein complexes in rice plasma membrane. Plant J 38:320-331

Kurakawa T, Ueda N, Maekawa M, Kobayashi K, Kojima M, Nagato Y, Sakakibara H, Kyozuka J (2007) Direct control of shoot meristem activity by a cytokininactivating enzyme. Nature 445:652-655

Li N, Li Y (2016) Signaling pathways of seed size control in plants. Curr Opin Plant Biol 33:23-32

Liu Q, Han R, Wu K, Zhang J, Ye Y, Wang S, Chen J, Pan Y, Li Q, Xu X, Zhou J, Tao D, Wu Y, Fu X (2018) G-protein betagamma subunits determine grain size through interaction with MADS-domain transcription factors in rice. Nat Commun 9:852

Mao H, Sun S, Yao J, Wang C, Yu S, Xu C, Li X, Zhang Q (2010) Linking differential domain functions of the GS3 protein to natural variation of grain size in rice. Proc Natl Acad Sci U S A 107:19579-19584

Miao J, Yang Z, Zhang D, Wang Y, Xu M, Zhou L, Wang J, Wu S, Yao Y, Du X, Gu F, Gong Z, Gu M, Liang G, Zhou Y (2019) Mutation of RGG2, which encodes a type $B$ heterotrimeric $G$ protein $\gamma$ subunit, increases grain size and yield production in rice. Plant Biotechnol J 17:650-664

Pandey S (2019) Heterotrimeric G-protein signaling in plants: conserved and novel mechanisms. Annu Rev Plant Biol 70:213-238

Robitaille M, Gora S, Wang Y, Goupil E, Pétrin D, Del Duca D, Villeneuve L, Allen B, Laporte S, Bernard D, Hébert T (2010) Gbetagamma is a negative regulator of AP-1 mediated transcription. Cell Signal 22:1254-1266

Stahl Y, Simon R (2010) Plant primary meristems: shared functions and regulatory mechanisms. Curr Opin Plant Biol 13:53-58

Sun H, Qian Q, Wu K, Luo J, Wang S, Zhang C, Ma Y, Liu Q, Huang X, Yuan Q, Han R, Zhao M, Dong G, Guo L, Zhu X, Gou Z, Wang W, Wu Y, Lin H, Fu X (2014) Heterotrimeric $G$ proteins regulate nitrogen-use efficiency in rice. Nat Genet 46:652-656

Sun S, Wang L, Mao H, Shao L, Li X, Xiao J, Ouyang Y, Zhang Q (2018) A Gprotein pathway determines grain size in rice. Nat Commun 9:851

Swain DM, Sahoo RK, Srivastava VK, Tripathy BC, Tuteja R, Tuteja N (2017) Function of heterotrimeric G-protein gamma subunit RGG1 in providing salinity stress tolerance in rice by elevating detoxification of ROS. Planta 245: 367-383

Thung L, Trusov Y, Chakravorty D, Botella JR (2012) G $1+$ 1+G $2+G \gamma 3=G \beta$ : the search for heterotrimeric G-protein $\gamma$ subunits in Arabidopsis is over. J Plant Physiol 169:542-545

Tsai YC, Weir NR, Hill K, Zhang W, Kim HJ, Shiu SH, Schaller GE, Kieber JJ (2012) Characterization of genes involved in cytokinin signaling and metabolism from rice. Plant Physiol 158:1666-1684

Ueguchi-Tanaka M, Fujisawa Y, Kobayashi M, Ashikari M, Iwasaki Y, Kitano H, Matsuoka M (2000) Rice dwarf mutant d1, which is defective in the alpha subunit of the heterotrimeric $\mathrm{G}$ protein, affects gibberellin signal transduction. Proc Natl Acad Sci U S A 97:11638-11643

Utsunomiya Y, Samejima C, Takayanagi Y, Izawa Y, Yoshida T, Sawada Y, Fujisawa $Y$, Kato H, Iwasaki $Y$ (2011) Suppression of the rice heterotrimeric $G$ protein beta-subunit gene, RGB1, causes dwarfism and browning of internodes and lamina joint regions. Plant J 67:907-916

Wang L, Xu Y-Y, Ma Q-B, Li D, Xu Z-H, Chong K (2006) Heterotrimeric G protein a subunit is involved in rice brassinosteroid response. Cell Res:14-20

Wang Y, Wu Y, Yu B, Yin Z, Xia Y (2017) EXTRA-LARGE G PROTEINs interact with E3 LIGases PUB4 and PUB2 and function in cytokinin and developmental processes. Plant Physiol 173:1235-1246

Wu Q, Xu F, Liu L, Char SN, Ding Y, Je Bl, Schmelz E, Yang B, Jackson D (2020) The maize heterotrimeric $G$ protein beta subunit controls shoot meristem development and immune responses. Proc Natl Acad Sci U S A 117:1799-1805

Xiao Y, Liu D, Zhang G, Gao S, Liu L, Xu F, Che R, Wang Y, Tong H, Chu C (2019) Big Grain3, encoding a purine permease, regulates grain size via modulating cytokinin transport in rice. J Integr Plant Biol 61:581-597

Yu Y, Assmann SM (2015) The heterotrimeric G-protein beta subunit, AGB1, plays multiple roles in the Arabidopsis salinity response. Plant Cell Environ 38: 2143-2156

Zhang D, Zhang M, Zhou Y, Wang Y, Shen J, Chen H, Zhang L, Lü B, Liang G, Liang J (2019) The rice $G$ protein $y$ subunit DEP1/qPE9-1 positively regulates grain-filling process by increasing auxin and cytokinin content in rice grains. Rice 12:91

Zhang DP, Zhou Y, Yin JF, Yan XJ, Lin S, Xu WF, Baluska F, Wang YP, Xia YJ, Liang GH, Liang JS (2015) Rice G-protein subunits QPE9-1 and RGB1 play distinct roles in abscisic acid responses and drought adaptation. J Exp Bot 66:6371-6384 
Zhou Y, Zhu J, Li Z, Yi C, Liu J, Zhang H, Tang S, Gu M, Liang G (2009) Deletion in a quantitative trait gene qPE9-1 associated with panicle erectness improves plant architecture during rice domestication. Genetics 183:315-324

Zong Y, Song Q, Li C, Jin S, Zhang D, Wang Y, Qiu J, Gao C (2018) Efficient C-to-T base editing in plants using a fusion of nCas9 and human APOBEC3A. Nat Biotechnol. https://doi.org/10.1038/nbt.4261

\section{Publisher's Note}

Springer Nature remains neutral with regard to jurisdictional claims in published maps and institutional affiliations.

\section{Submit your manuscript to a SpringerOpen ${ }^{\mathcal{O}}$ journal and benefit from:}

- Convenient online submission

- Rigorous peer review

- Open access: articles freely available online

- High visibility within the field

- Retaining the copyright to your article

Submit your next manuscript at $\boldsymbol{\nabla}$ springeropen.com 